\title{
CEO-Endodontia da UFRGS: um estudo transversal sobre a prevalência de atendimentos, características dos pacientes e documentação dos prontuários
}

Mariana Travi Pandolfo*; Jessye Melgarejo do Amaral Giordani**; Matheus Neves***; Renata Grazziotin Soares****

\author{
Pós-graduanda do Curso de Especialização em \\ Atenção Especializada em Saúde, ênfase em \\ Endodontia, Universidade Federal do Rio Grande do \\ Sul \\ ** Doutorando do Programa de Pós-graduação em \\ Epidemiologia, Universidade Federal do Rio Grande \\ do Sul \\ *** Doutorando em Odontologia, área de concentração \\ em Saúde Bucal Coletiva, Universidade Federal do \\ Rio Grande do Sul \\ **** Professora Adjunta da Faculdade de Odontologia, \\ Universidade Federal do Rio Grande do Sul
}

\section{RESUMO}

O presente estudo sobre tratamento de canais radiculares teve como objetivos: i) fornecer um panorama de atendimentos dos pacientes referenciados pelas Unidades Básicas de Saúde (UBS) de Porto Alegre no CEO da Universidade Federal do Rio Grande do Sul (UFRGS) e ii) investigar a conformidade de preenchimento dos prontuários clínicos dos pacientes atendidos. Foram analisados 242 prontuários. Os dados coletados foram inseridos em uma tabela com as seguintes informações referentes a cada paciente: 1) número do prontuário, 2) idade (anos $\mathrm{e}$ meses), 3) gênero (masculino/feminino), 4) dente, 5) número de consultas, 6) presença de radiografia final do dente obturado, restaurado e sem isolamento absoluto (sim/não), 7) qualidade da radiografia (adequada/ inadequada), 8) presença da radiografia final do caso no momento da obturação ( $\operatorname{sim} /$ não), 9) qualidade da radiografia (adequada/ inadequada), 10) consentimento informado assinado pelo paciente (sim/não), 11) entrevista dialogada preenchida (sim/não), 12) presença da informação sobre o diagnóstico pulpar ou periapical (sim/não), 13) relato da finalização do tratamento/obturação do dente (sim/não), 14) relato sobre a restauração provisória coronária ( $\operatorname{sim} /$ não), 15) informação sobre a contrareferência para a UBS (sim/não). Foi realizada análise descritiva (frequência e percentagem) dos dados e correlação entre as variáveis idade (faixa etária) e i) gênero, ii) dente (posterior ou anterior), iii) grupo dentário e iv) número de consultas. Houve predominância de pacientes do sexo feminino $(63,2 \%)$ em todas as faixas etárias, exceto em pacientes com 60 anos ou mais, onde predominaram pacientes do sexo masculino. Os dentes que mais sofreram intervenção foram os posteriores $(60,3 \%)$, exceto em pacientes na faixa etária 50 a 59 anos. Os $1^{\text {os }}$ molares foram os dentes que receberam mais tratamento endodôntico $(28,9 \%)$, principalmente em pacientes 
adultos-jovens (menores de 19 anos até 39 anos de idade). Na maioria dos casos o tratamento foi concluído em poucas consultas (67,4\% das vezes em até 3 consultas). Os pontos negativos observados foram: deficiente armazenamento e qualidade das radiografias; ausência da história médicoodontológica do paciente; falta da descrição do diagnóstico pulpar/periapical e ausência da assinatura do paciente autorizando o tratamento. Os pontos positivos encontrados foram: a finalização do tratamento endodôntico foi documentada em $100 \%$ das vezes e a realização da restauração provisória coronária foi documentada na maioria das vezes $(95 \%)$. Os achados deste estudo, além de evidenciar o perfil de atendimento do CEOEndodontia da UFRGS, podem contribuir para o desenvolvimento de estratégias que impulsionem mudanças positivas no serviço odontológico, tais como incentivo do pensamento crítico e ético no aluno/profissional em relação à magnitude da importância do correto preenchimento dos prontuários clínicos e geração de ideias que possam aprimorar os meios de armazenamento das radiografias nos prontuários.

Descritores: Prontuário. Endodontia. Centro de Especialidades Odontológicas.

\section{INTRODUÇÃO}

A prestação de serviços públicos de saúde bucal no Brasil sempre foi caracterizada por ações de baixa complexidade, em sua maioria curativas e mutiladoras, com uma abrangência bastante restrita. Nos últimos anos, porém, conforme os dados da Política Nacional de Saúde Bucal (PNSB), o acesso dos brasileiros aos serviços públicos odontológicos melhorou consideravelmente, muito embora ainda não tenham sido completamente solucionados os problemas relacionados ao acesso aos serviços especializados ${ }^{1}$.

Diante desse cenário, foi instituída a
Política Nacional de Saúde Bucal - Programa Brasil Sorridente - pelo Ministério da Saúde², visando garantir as ações de promoção, prevenção e recuperação da saúde bucal da população. Com base nessa Política, foram criadas unidades de referência para a Atenção Básica em todas as regiões brasileiras, chamadas Centro de Especialidades Odontológicas (CEO), que têm o desafio de ampliar e qualificar a oferta de serviços odontológicos especializados.

Os CEOs são estabelecimentos de saúde bucal, participantes do Cadastro Nacional de Estabelecimentos de Saúde (CNES), que oferecem serviços nas especialidades de Endodontia, Periodontia e Estomatologia (com ênfase no diagnóstico e detecção do câncer de boca). Esses estabelecimentos atuam como unidades de referência para as Equipes de Saúde Bucal da Atenção Básica, tendo como principal função realizar procedimentos especializados e contra-referenciar os usuários para que os profissionais das Unidades Básicas de Saúde concluam os tratamentos.

Considerando os significativos desafios quanto à integralidade do Sistema Único de Saúde (SUS) e à construção de uma rede efetiva e articulada de atenção à saúde bucal, identificou-se a necessidade de formação de profissionais capazes de gerir os serviços de saúde bucal com maior conhecimento das especificidades da rede SUS. A partir de então, foi celebrado um convênio entre a Universidade Federal do Rio Grande do Sul (UFRGS) e o Ministério da Saúde para a realização do Curso de Atenção Especializada em Saúde, com ênfases nas áreas de Endodontia, Periodontia, Cirurgia, Estomatologia e Gestão, objetivando a formação de profissionais capacitados e qualificados para atuarem nesses serviços 
públicos especializados em saúde bucal. Em virtude da área de Endodontia constituir um dos campos de atuação estratégicos para o SUS, o presente estudo forneceu um panorama sobre os atendimentos de pacientes realizados no CEO de Endodontia da UFRGS. Além disso, objetivando otimizar a formação do estudante de pós-graduação no que concerne aos aspectos éticos e legais da profissão, investigou-se a conformidade de preenchimento dos prontuários clínicos dos pacientes atendidos.

\section{METODOLOGIA}

O presente estudo de delineamento transversal foi aprovado na Comissão de Pesquisa da UFRGS em 15/08/2014, conforme a Ata n 09/2014, e no Comitê de Ética em Pesquisa (CEP), em 16/10/2014, conforme o Certificado de Apresentação para Apreciação Ética (CAAE) n 37110914. 3.0000.5347, sendo realizado por meio de coleta de informações em prontuários de pacientes do CEO - Endodontia UFRGS, atendidos pelos alunos do Curso de Especialização em Atenção Especializada em Saúde.

A amostra foi constituída pela documentação dos pacientes atendidos no período compreendido entre fevereiro e dezembro de 2014, totalizando 242 tratamentos endodônticos realizados.

Os dados coletados foram registrados em instrumento específico da pesquisa, contendo as seguintes informações: 1) número do prontuário, 2) idade (anos e meses), 3) gênero (masculino/feminino), 4) dente, 5) número de consultas, 6) presença de radiografia final do dente obturado, restaurado e sem isolamento absoluto ( $\operatorname{sim} /$ não), 7 ) qualidade da radiografia (adequada/ inadequada), 8) presença da radiografia final do caso no momento da obturação (sim/não), 9) qualidade da radiografia (adequada/ inadequada), 10) consentimento informado assinado pelo paciente (sim/não), 11) entrevista dialogada preenchida (sim/não), 12) presença da informação sobre o diagnóstico pulpar ou periapical (sim/não), 13) relato da finalização do tratamento/ obturação do dente (sim/não), 14) relato sobre a restauração provisória coronária (sim/não), 15) informação sobre a contra-referência para a UBS (sim/não).

A análise descritiva (frequência e a percentagem) das variáveis coletadas foi realizada a partir da tabulação dos dados no software Microsoft ${ }^{\circledR}$ Excel $^{\circledR}$ para Mac 2011 [Versão 14.4.7(141117)].

Posteriormente, a variável idade (faixa etária) foi correlacionada com i) gênero, ii) dente (posterior ou anterior), iii) grupo dentário e iv) número de consultas. Para isso, foi utilizado o programa SPSS (Statistical Package for The Social Sciences versão 16.0 [SPSS Inc. Chicago, IL]).

\section{RESULTADOS}

A amostra foi constituída por 242 pacientes; sendo 153 do gênero feminino $(63,2 \%)$ e de 89 do gênero masculino (36,8\%). Em relação à idade dos pacientes, a frequência e percentagem de pacientes que foram submetidos à tratamento endodôntico de acordo com as faixas etárias foi o seguinte: os pacientes de 30 a 39 anos estiveram presentes em maior número $(n=60 ; 24,8 \%)$; seguidos por indivíduos de 40 a 49 anos ( $\mathrm{n}=52 ; 21,5 \%)$; pacientes de até 19 anos ( $\mathrm{n}=43,17,8 \%) ; 20$ a 29 anos $(\mathrm{n}=34,14 \%) ; 50$ a 59 anos $(\mathrm{n}=32$, $13,2 \%)$ e, pacientes com 60 anos ou mais $(\mathrm{n}=21,8,7 \%)$. A tabela 1 mostra a correlação entre a faixa etária do paciente e o gênero, evidenciando que os pacientes do gênero 
masculino predominaram apenas na faixa etária maior que 60 anos $(n=16 ; 76,2 \%)$.

Os dentes que mais sofreram intervenção endodôntica foram os dentes posteriores $(n=146 ; 60,3 \%)$, em relação aos anteriores $(n=87 ; 36 \%)$. Em 9 casos não havia relato sobre qual o dente tratado $(3,7 \%)$. A tabela 2 mostra a correlação entre a faixa etária do paciente e a frequência de tratamento endodôntico em dentes anteriores e posteriores. É interessante notar que em todas as faixas etárias, exceto em pacientes de 50 a 59 anos, os dentes posteriores foram os que mais sofreram tratamento endodôntico.

No que concerne aos grupos dentários, os dentes que mais receberam tratamento endodôntico foram os $1^{\text {os }}$ molares $(\mathrm{n}=70$; $28,9 \%)$, seguido por incisivos $(\mathrm{n}=66 ; 27,3 \%)$; pré-molares $(\mathrm{n}=51 ; 21,1 \%) ; 2^{\text {os }}$ molares $(\mathrm{n}=25 ; 10,3 \%)$ e, por último, caninos $(\mathrm{n}=21$; $8,7 \%$ ). Este valor totaliza 233 dentes, já que em 9 prontuários não havia o relato de qual o dente que sofreu intervenção. Após correlacionar-se o grupo dentário com a faixa etária do paciente (tabela 3) é possível discernir os diferentes padrões de necessidade endodôntica: as três primeiras faixas etárias estudadas (menores de 19 anos até 39 anos de idade) necessitaram mais tratamento endodôntico nos $1^{\text {os }}$ molares; porém, nas faixas etárias seguintes este padrão não se manteve, havendo similar necessidade de tratamento também em pré-molares e dentes anteriores.

Em relação ao número de consultas, observou-se que dos 242 pacientes, 192 deles necessitaram de até três consultas $(79,3 \%)$ e 50 pacientes necessitaram de mais de três consultas $(20,7 \%)$. Na maioria dos casos, o profissional utilizou duas consultas para conclusão do tratamento, conforme observase a seguir: uma consulta $(n=8 ; 3,3 \%)$, duas consultas $(\mathrm{n}=109,45 \%)$, três consultas $(\mathrm{n}=75$; $31 \%)$, quatro consultas $(\mathrm{n}=26 ; 10,7 \%)$, cinco consultas $(n=14 ; 5,8 \%)$, seis consultas $(n=4$; $1,7 \%)$, sete consultas $(n=4 ; 1,7 \%)$, oito consultas $(n=1 ; 0,4 \%)$ e, finalmente, dez consultas $(n=1 ; 04 \%)$. Após correlacionar-se o número de consultas para finalização do tratamento com as faixas etárias dos pacientes (tabela 4) observa-se que para todas as faixas etárias, a minoria dos casos necessitou de mais de 3 consultas. O mesmo ocorreu para os pacientes $\geq 19$ anos de idade, porém, nessa faixa etária ficou evidente um aumento na necessidade de maior número de consultas (até três consultas: $n=29,67,4 \%$; mais de três consultas: $n=14,32,6 \%$ ).

O diagnóstico pulpar e periapical não constava no prontuário na grande maioria dos casos $(n=207 ; 85,5 \%)$. No restante dos casos $(\mathrm{n}=35 ; 14,5 \%)$ o diagnóstico apresentado foi: dente vital $(n=4 ; 1,7 \%)$; necrose sem lesão periapical $(\mathrm{n}=7 ; 2,9 \%)$; necrose com lesão periapical $(n=23 ; 9,5 \%)$ e lesão endoperiodontal $(\mathrm{n}=1 ; 0,4 \%)$.

A radiografia final (no momento da obturação, ainda com isolamento absoluto) constava no prontuário do paciente em aproximadamente metade da amostra $[\operatorname{sim}=122(50.4 \%) ;$ não=120 (49,6\%)]. Se presente, a qualidade desta radiografia foi classificada como adequada em 42,6\% dos casos. A radiografia final (com o dente obturado e restaurado, porém já sem isolamento absoluto) estava presente em 154 casos $(63,3 \%)$, sendo adequada em $52,9 \%$ das vezes.

O relato sobre a finalização do tratamento de canal radicular foi documentado em $100 \%(\mathrm{n}=242)$ das vezes. A realização da restauração provisória coronária, após finalização do tratamento endodôntico, foi documentada na maioria das 
vezes [ $\operatorname{sim}=230(95 \%)$; não=12 (5\%)].

$\mathrm{O}$ questionário de saúde relativo à história médico-odontológica do paciente estava preenchido em 211 (87,2\%) prontuários, estando ausente em 31 (12,8\%). $\mathrm{O}$ consentimento informado autorizando a realização do tratamento e dando ciência sobre os riscos inerentes estava assinado em 239 (98.8\%) prontuários; apenas em $3(1,2 \%)$ não constava a assinatura do paciente. A informação sobre a contra-referência do paciente para a UBS esteve presente em 160 $(66,1 \%)$ dos prontuários e ausente em 82 $(33,9 \%)$.

\section{DISCUSSÃO}

A importância de se investigar o perfil dos pacientes atendidos em determinado serviço de saúde, bem como avaliar a organização dos prontuários clínicos adicionado à escassez de estudos dirigidos aos serviços de saúde que participam do CEO de Endodontia foram os principais fatores que motivaram esse trabalho.

De maneira geral, os resultados obtidos neste estudo sugerem que o perfil dos pacientes atendidos no CEO de Endodontia da UFRGS é similar a outros serviços de saúde ${ }^{3}$, e que existe uma razoável inserção de informações nos prontuários clínicos, apesar da grande ausência dos exames de imagem e da negligência em relação à documentação do diagnóstico pulpar/periapical.

Em relação ao gênero, houve predominância de pacientes do sexo feminino $(63,2 \%)$. Isso pode ser justificado em razão de as mulheres serem maioria no Brasil, e também por se preocuparem mais com a saúde e estética ${ }^{4}$. Além disso, as mulheres, por acompanharem os filhos e os idosos nas consultas, tornam-se naturalmente prédispostas a frequentar serviços de saúde 5 .
Nessa linha de pensamento, pode-se pressupor que o maior número de pacientes do sexo feminino atendidos no CEO de Endodontia está de acordo, provavelmente, com o maior número de pacientes do gênero feminino também nas outras clínicas do curso de graduação da UFRGS ${ }^{6}$.

Este achado está de acordo com outros estudos que também retratam o perfil de atendimento odontológico no Brasil, como por exemplo, no estudo de Reis et al. ${ }^{3}$, no qual os autores evidenciaram porcentagem maior de pacientes do sexo feminino atendidos $(67,8 \%)$ nas Clínicas Integradas da Faculdade de Odontologia da Universidade Federal de Goiás. Após associar-se a faixa etária com o gênero (tabela 1) foi possível obter um panorama mais detalhado sobre as características dos pacientes do CEOEndodontia da instituição: o acolhimento de pacientes jovens ( $\geq 19$ anos de idade) pode ser considerado semelhante tanto para mulheres $(48,8 \%)$ como para homens $(51,2 \%)$; a partir dessa idade há predominância de pacientes do sexo feminino; e, por fim, há mais homens idosos ( $\leq 60$ anos de idade) sendo atendidos. Estes achados são particularmente importantes pois sugerem que os alunos/profissionais que integram o CEOEndodontia da UFRGS devem estar preparados para atender pacientes das diferentes faixas etárias, sempre tentando entender o contexto sócio-econômico-cultural que o mesmo vive, provendo, dessa maneira, o melhor acolhimento possível.

Os achados do presente estudo também demonstraram que os dentes molares foram os que mais receberam tratamento endodôntico. Isso provavelmente se deve ao fato desses dentes possuírem anatomia mais complexa $^{7,8}$, dificultando a sua higienização, e também pela falta de instrução de higiene a 
Tabela 1 - Correlação (frequência e percentagem) entre faixa etária e gênero

\begin{tabular}{|c|c|c|c|c|c|c|c|c|}
\hline \multirow{5}{*}{ Gênero } & & \multicolumn{7}{|c|}{ Faixa Etária (anos) } \\
\hline & & $\geq 19$ & $20-29$ & $30-39$ & $40-49$ & $50-59$ & $\leq 60$ & Total \\
\hline & $\mathrm{F}$ & $21(48,8 \%)$ & $26(76,5 \%)$ & $44(73,3 \%)$ & $32(61,5 \%)$ & $25(78,1 \%)$ & $5(23,8 \%)$ & $153(63,2 \%)$ \\
\hline & M & $22(51,2 \%)$ & $8(23,5 \%)$ & $16(26,7 \%)$ & $20(38,5 \%)$ & $7(21,9 \%)$ & $16(76,2 \%)$ & $89(36,8 \%)$ \\
\hline & Total & $43(100 \%)$ & $34(100 \%)$ & $60(100 \%)$ & $52(100 \%)$ & $32(100 \%)$ & $21(100 \%)$ & $242(100 \%)$ \\
\hline
\end{tabular}

F: feminino, M: masculino

Tabela 2 - Correlação (frequência/percentagem) entre faixa etária e tratamento endodôntico em dentes anteriores e posteriores

\begin{tabular}{ccccccccc}
\hline \multicolumn{7}{c}{ Faixa Etária (anos) } \\
\cline { 3 - 9 } Dente & A & $13(32,5 \%)$ & $10(30,3 \%)$ & $15(25,4 \%)$ & $21(42,0 \%)$ & $18(60,0 \%)$ & $10(47,6 \%)$ & $87(37,3 \%)$ \\
& $\mathrm{P}$ & $27(67,5 \%)$ & $23(69,7 \%)$ & $44(74,6 \%)$ & $29(58,0 \%)$ & $12(40,0 \%)$ & $11(52,4 \%)$ & $146(62,7 \%)$ \\
& Total & $40(100 \%)$ & $33(100 \%)$ & $59(100 \%)$ & $50(100 \%)$ & $30(100 \%)$ & $21(100 \%)$ & $233(100 \%)$ \\
\hline
\end{tabular}

A: anterior, P: posterior

Tabela 3 - Correlação entre a faixa etária do paciente e a frequência/percentagem de tratamento endodôntico nos diferentes grupos dentários

\begin{tabular}{ccccccccc}
\hline \multicolumn{7}{c}{} & \multicolumn{7}{c}{ Faixa Etária (anos) } \\
\cline { 3 - 9 } Grupo & $\mathrm{I}$ & $13(32,5 \%)$ & $9(27,3 \%)$ & $15(25,4 \%)$ & $14(28,0 \%)$ & $12(40,0 \%)$ & $3(14,3 \%)$ & $66(28,3 \%)$ \\
Dentário & $\mathrm{C}$ & 0 & $1(3,0 \%)$ & 0 & $7(14,0 \%)$ & $6(20,0 \%)$ & $7(33,3 \%)$ & $21(9,0 \%)$ \\
& $\mathrm{PM}$ & $2(5,0 \%)$ & $6(18,2 \%)$ & $15(25,4 \%)$ & $18(36,0 \%)$ & $3(10,0 \%)$ & $7(33,3 \%)$ & $51(21,9 \%)$ \\
& $1^{\circ} \mathrm{M}$ & $24(60,0 \%)$ & $10(30,3 \%)$ & $22(37,3 \%)$ & $7(14,0 \%)$ & $5(16,7 \%)$ & $2(9,5 \%)$ & $70(30,0 \%)$ \\
& $2^{\circ} \mathrm{M}$ & $1(2,5 \%)$ & $7(21,2 \%)$ & $7(11,9 \%)$ & $4(8,0 \%)$ & $4(13,3 \%)$ & $2(9,5 \%)$ & $25(10,7 \%)$ \\
& Total & $40(100 \%)$ & $33(100 \%)$ & $59(100 \%)$ & $50(100 \%)$ & $30(100 \%)$ & $21(100 \%)$ & $233(100 \%)$ \\
\hline
\end{tabular}

I: incisivos, C: caninos, PM: pré-molares, M: molares

Tabela 4 - Correlação entre o número de consultas para finalização do tratamento e faixa etária.

\section{Faixa Etária (anos)}

\begin{tabular}{rcccc|r|rrr}
\cline { 3 - 9 } Consultas & & $\geq 19$ & $20-29$ & $30-39$ & $40-49$ & \multicolumn{1}{c}{$50-59$} & $\leq 60$ & \multicolumn{2}{c}{ Total } \\
& Até 3 & $29(67,4 \%)$ & $27(79,4 \%)$ & $49(81,7 \%)$ & $43(82,7 \%)$ & $26(81,3 \%)$ & $18(85,7 \%)$ & $192(79,3 \%)$ \\
& $>3$ & $14(32,6 \%)$ & $7(20,6 \%)$ & $11(18,3 \%)$ & $9(17,3 \%)$ & $6(18,8 \%)$ & $3(14,3 \%)$ & $50(20,7 \%)$ \\
& Total & $43(100 \%)$ & $34(100 \%)$ & $60(100 \%)$ & $52(100 \%)$ & $32(100 \%)$ & $21(100 \%)$ & $242(100 \%)$ \\
\hline
\end{tabular}


esses pacientes no passado. Além disso, (apesar de que a etiologia da necessidade de tratamento endodôntico não ter sido escopo deste estudo), restaurações dentárias deficientes podem ter levado esses pacientes a necessitarem de tratamento endodôntico.

Os resultados em relação aos grupos dentários tratados endodonticamente e a faixa etária do paciente refletem as condições de saúde bucal no Brasil, evidenciando a grande necessidade de tratamento odontológico em pacientes jovens ${ }^{9}$.

Em relação ao número de consultas, na grande parte das vezes o profissional necessitou de duas consultas para finalizar o tratamento, sendo que para um dos usuários foram necessárias dez consultas. Esse fato pode ser considerado uma desvantagem para um CEO, que deve realizar uma produção mínima mensal, definida na Portaria 1.464/GM, de 20117. Outro aspecto importante é o significativo custo para o tratamento de apenas um dente.

A análise de correlação entre a faixa etária que o paciente se enquadra e o número de consultas necessárias é interessante pois mostra que pacientes muito jovens ( $\geq 19$ anos de idade) necessitaram de mais de 3 consultas em $32,6 \%$ das vezes. Este dado pode sugerir que os estudantes/profissionais tiveram, relativamente, mais dificuldade de manejo destes pacientes, optando, talvez, por consultas mais curtas e em maior número.

No que concerne aos resultados do diagnóstico pulpar e periapical a necrose com lesão periapical foi a ocorrência mais frequente. Este resultado não evidencia realmente qual a situação pulpar/periapical dos dentes tratados no CEO-Endodontia da UFRGS, já que a grande maioria dos prontuários $(85,5 \%)$ não mencionava o diagnóstico. A falta da informação sobre o diagnóstico pulpar/periapical é um grande problema para o profissional que está prestando serviço, bem como para os demais cirurgiões-dentistas que porventura necessitem atender o mesmo paciente. Em situações de emergência, por exemplo, é preciso tomar a decisão clínica de acordo com o diagnóstico inicial e, também, conforme os procedimentos que foram anteriormente realizados no transoperatório. Em outras palavras, um determinado diagnóstico vai guiar a escolha da imediata reintervenção, da solução irrigadora, da medicação intracanal, da medicação sistêmica, entre outros fatores importantes, tais como a possibilidade de obturação e o período e intervalo para controle pós-operatório.

Outro tópico de extrema relevância são os problemas jurídicos associados ao incorreto preenchimento do prontuário e anexação da documentação do paciente. Para um prontuário clínico ser considerado adequado legalmente, ele deve apresentar toda a documentação padronizada e destinada ao registro de informações pertinentes ao usuário do serviço, do início ao final do tratamento. Normas éticas e legais devem nortear o correto preenchimento dos prontuários, pois falhas na documentação poderão gerar conflitos futuros ${ }^{10}$. Dessa forma, o prontuário deve conter todos os procedimentos propostos (plano de tratamento) e, também, os procedimentos realizados, devidamente assinados pelo paciente e profissional, sem rasuras ou abreviações. É justamente no cuidadoso preenchimento do prontuário que o cirurgião-dentista encontra $o$ fator mais importante na prevenção de problemas jurídicos ${ }^{11}$.

Nesse contexto, foram analisados no estudo os dados referentes às radiografias e ao correto preenchimento dos prontuários (relato 
sobre a finalização do tratamento de canal radicular, questionário de saúde e assinatura do consentimento informado). Os resultados em relação às radiografias demonstraram que a radiografia final (no momento da obturação) constava no prontuário do paciente em aproximadamente metade da amostra $(50,4 \%)$, e quando presente, a radiografia apresentava qualidade adequada em menos que a metade das vezes. Por outro lado, a radiografia final (com o dente obturado e restaurado) estava presente em um pouco mais da metade dos prontuários analisados $(63,3 \%)$ e, nesse caso, se apresentava adequada apenas na metade das vezes. Estes dados são, lamentavelmente, um grande problema que atinge tanto o paciente que recebeu o cuidado, como o profissional e, também, a instituição. Acredita-se que inúmeras são as causas do deficiente armazenamento das radiografias (negligência, falta de condições físicas de armazenamento, necessidade de meios digitais de condicionamento das informações), fatores que nos permitem dizer que o CEO-Endodontia da UFRGS clama por uma melhoria urgente nesse aspecto. Novamente, é importante ressaltar que, em processos éticoadministrativos ou judiciais, as radiografias são os instrumentos de prova mais importantes para a comprovação dos tratamentos realizados ${ }^{12}$. Portanto, associada à finalidade clínica, a documentação radiográfica pode constituir uma relevante fonte de prova em questões periciais, mas para subsidiarem efetivamente os processos judiciais, as radiografias devem apresentar-se com uma boa qualidade ${ }^{13}$.

Outros pontos negativos relevantes identificados a partir da análise dos dados foram a ausência das informações relativas à história médico odontológica em 31 prontuários $(12,8 \%)$ e a falta de assinatura do paciente no texto referente ao consentimento informado em três prontuários (1,2\%). Em relação à falta da assinatura do paciente, é importante ter clareza que no inciso XII do art. $7^{\circ}$ do Código de Ética Odontológica ${ }^{10}$, há previsão de infração ética a quem iniciar qualquer procedimento sem o consentimento prévio do paciente ou do seu responsável legal, exceto em casos de urgência e emergência. Cabe ainda ressaltar que o termo de consentimento informado não exclui o profissional de suas responsabilidades e de futuro processo judicial, devendo ser elaborado de forma clara e com linguagem acessível $^{10}$.

$\mathrm{O}$ relato sobre a finalização do tratamento de canal radicular foi documentado em todos os prontuários analisados. Esse dado é de suma importância e foi um achado positivo desta presente pesquisa. Juntamente com a documentação radiográfica, o relato da finalização do tratamento também é utilizado na defesa do profissional frente à ação judicial, além de servir para constatação da evolução clínica do caso a longo prazo ${ }^{10}$. Além disso, é importante lembrar que o detalhamento por escrito da finalização da endodontia (data, sintomatologia, sinais clínicos, tipo de restauração provisória etc.), também deve ser reportado no documento de contra-referência para a UBS de origem. Essas informações são fundamentais para o cirurgião-dentista da rede de atenção primária, pois assim ele terá todas as informações necessárias para dar continuidade ao tratamento daquele usuário.

\section{CONCLUSÃO}

Este estudo caracterizou os pacientes atendidos no CEO-Endodontia da UFRGS mostrando que eles são, na maioria do sexo feminino em todas as faixas etárias, exceto em 
pacientes com 60 anos ou mais, faixa de idade onde predominou o sexo masculino. Os dentes que mais sofreram intervenção foram os posteriores $(60,3 \%)$, exceto em pacientes na faixa etária de 50 a 59 anos. Os $1^{\text {os }}$ molares foram os dentes que receberam mais tratamento endodôntico (28,9\%), principalmente em pacientes adultos-jovens (menores de 19 anos até 39 anos de idade). Ainda, observou-se que os profissionais/alunos concluíram os tratamentos de seus pacientes em poucas consultas (em $67,4 \%$ das vezes em até 3 consultas), o que é um indício de adequado conhecimento e produtividade; mas falharam em relação a quatro importantes tópicos: armazenamento e qualidade dos exames de imagem; descrição da história médico-odontológica do paciente; descrição sobre o diagnóstico pulpar/periapical e assinatura do paciente autorizando o tratamento.

Os achados deste estudo, além de evidenciar o perfil de atendimento do CEOEndodontia da instituição, podem contribuir para o desenvolvimento de estratégias que impulsionem mudanças positivas no serviço odontológico, tais como incentivo do pensamento crítico e ético no aluno/ profissional em relação à magnitude da importância do correto preenchimento dos prontuários clínicos, bem como da sua adequada documentação radiográfica e geração de ideias que possam aprimorar os meios de armazenamento das radiografias nos prontuários.

\section{ABSTRACT \\ CEO-endodontics at UFRGS: a cross sectional study on the prevalence of care, patient characteristics and documentation of clinical records}

This study on root canal treatment aimed: i) to provide an overview regarding the treatment of patients referred by the Basic Health Units (UBS) of Porto Alegre at the CEO of the Universidade Federal do Rio Grande do Sul (UFRGS) and ii) to investigate the adequate fulfillment of the patient's clinical records. Clinical records from 242 patients were analyzed. Data were recorded into a table with the following information for each patient: 1) clinical record number, 2) age (years and months), 3) gender (male / female), 4) tooth, 5) number of appointments 6) presence of the final radiograph showing the root canal filling and the tooth restored without the rubber dam (yes / no), 7) quality of the radiography (adequate / inadequate), 8) presence of the final radiography at the time of root canal filling (yes / no), 9) quality of radiography (adequate / inadequate), 10) informed consent signed by the patient (yes / no), 11) fulfilment of the dialogue-interview (yes / no), 12) presence of the information about the pulp or periapical diagnosis (yes / no ), 13) report the completion of treatment / filling of the tooth (yes / no), 14) report on coronary temporary restoration (yes / no), 15) information on the counter-reference to the UBS (yes / no). The descriptive analysis (frequency and percentage) and correlation between the variables age (age group) with: i) gender, ii) tooth (anterior or posterior), iii) dental group and iv) number of appointments were performed. There was a predominance of female patients (63.2\%) in all age groups, except in patients 60 years or older, where men were predominant. The majority of the treatments were in posterior teeth $(60,3 \%)$, except in patients aged 50-59 years. The 1st molars were the teeth with more endodontic treatment $(28.9 \%)$, mainly in young adults patients (younger than 19 years up to 39 years old). Most treatments were completed in fewer appointments (in $67.4 \%$ of the cases up to 3 consultations). The negative points observed were: poor storage and quality of radiographs, lack of medical and dental history of the patient, lack of description on the pulp/periapical diagnosis and absence of the patient's signature authorizing the 
treatment. The positives points were: the written report regarding the treatment completion was present in $100 \%$ of the cases, and the report regarding the coronary temporary restoration was documented in the most of the cases $(95 \%)$. The findings of this present study described the service characteristics of the CEO-Endodontics at UFRGS. It also may contribute to the development of strategies that stimulate positive changes in the dental service, such as fostering students' critical and ethical thinking regarding the magnitude of importance to report correctly the patients' clinical records, and to generate ideas to improve the storage of the radiographs in the patients' clinical records.

Descriptors: Patient's Clinical Record. Endodontics. Specialized Dental Center.

\section{REFERÊNCIAS}

1. Nassri MRG, Silva AS, Yoshida AT. Levantamento do perfil socioeconômico de pacientes atendidos na clínica odontológica da Universidade de Mogi das Cruzes e do tratamento ao qual foram submetidos: clínica endodôntica. Rev Sul-Bras Odontol. 2009; 6(3):272-8.

2. Ministério da Saúde. Diretrizes da Política Nacional de Saúde Bucal. Brasília: Ministério da Saúde; 2004. Disponível em: http://189.28.128.100/ dab/docs/publicacoes/geral/diretrizes_da politica_nacional_de_saude_bucal.pdf

3. Reis SCGB, Santos LB, Leles CR. Clínica Integrada de ensino Odontológico: Perfil dos Usuários e Necessidades Odontológicas. Rev Odontol Bras Central. 2011;20(52):4651.

4. Tortamano IP, Leopoldino VD, Borsatti MA, Penha SS, Buscariolo IA, Costa CG, Rocha RG. Aspectos epidemiológicos e sociodemográficos do Setor de Urgência da Faculdade de Odontologia da
Universidade de São Paulo. Rev Pós Grad. 2007;13(4):299-306.

5. Sanchez HF, Drumond MM. Atendimento de urgências em uma Faculdade de Odontologia de Minas Gerais: perfil do paciente e resolutividade. RGO. 2011; 59(1):79-86.

6. Andriola FO, Toassi RFC, de Paris MF, Baraldi CEE, Freddo AL. Perfil sóciodemográfico, epidemiológico e comportamental dos pacientes atendidos no ambulatório de exodontia da FO-UFRGS e a efetividade dos atendimentos realizados. Arq Odontol. 2015;51(2):104-15.

7. Ministério da Saúde. Portaria $\mathrm{n}^{\mathrm{o}}$ 1.464/GM de 24 de junho de 2011. Altera o anexo da Portaria $n^{\circ}$ 600/MG/MS, de 23 de março de 2006, que institui o financiamento dos Centros de Especialidades Odontológicas (CEO). Brasília: Ministério da Saúde; 2011. Disponível em: http://bvsms.saude.gov. br/bvs/saudelegis/gm/2011/prt1464_24_ 06_2011.html

8. Zhang R, Yang H, Yu X, Wang H, Hu T, Dummer PM. Use of CBCT to identify the morphology of maxillary permanent molar teeth in a Chinese subpopulation. Int Endod J. 2011;44:162-9.

9. SB Brasil 2010. Coordenação Nacional de Saúde Bucal, Ministério da Saúde. Condições de saúde bucal da população brasileira. Resultados principais. Disponível em: http://dab.saude.gov.br/ CNSB/sbbrasil/arquivos/projeto_sb2010 relatorio_final.pdf

10. Costa SM, Braga SL, Abreu MHNG, Bonan PRF. Questões éticas e legais no preenchimento das fichas clínicas odontológicas. RGO. 2009;57(2):211-6.

11. Benedicto EM, Lages LHR, Oliveira OF, Silva RHA, Paranhos LR. A importância 
da correta elaboração do prontuário odontológico. Odonto (São Bernardo do Campo). 2010; 18(36):41-50.

12. Ditterich RG, Portero PP, Grau P, Rodrigues CK, Wambier DS. A importância do prontuário odontológico na clínica de graduação em Odontologia e a responsabilidade ética pela sua guarda. Rev Inst Ciênc Saúde. 2008; 26(1):120-4.
13. Lima LR, Lima Júnior GTA, Machado Filho JA, Freitas SAP. Avaliação da qualidade e do arquivamento de radiografias periapicais na clínica de endodontia da Faculdade NOVAFAPI. Odontol Clín-Cient. 2010;9(4):355-8.

Correspondência para:

Mariana Pandolfo

e-mail: marianapandolfo@gmail.com

Faculdade de Odontologia da UFRGS

Rua Ramiro Barcelos, 2492

90035-004 - Porto Alegre, RS 